\title{
PERIODIC SOLUTIONS OF NONLINEAR VIBRATING STRINGS AND DUALITY PRINCIPLES
}

\author{
BY HAÏM BREZIS
}

Introduction. Our lecture deals with the study of $T$-periodic solutions for the nonlinear vibrating string equation:

$$
\begin{array}{ll}
u_{t t}-u_{x x}+g(u)=f(x, t), & 0<x<\pi, t \in \mathbf{R}, \\
u(x, t)=0, & x=0, x=\pi, t \in \mathbf{R}, \\
u(x, t+T)=u(x, t), & 0<x<\pi, t \in \mathbf{R} .
\end{array}
$$

Here $g$ denotes a continuous function on $\mathbf{R}$ such that $g(0)=0$ and $f(x, t)$ is a given $T$-periodic function of $t$.

Problem (1) may be viewed as an infinite-dimensional Hamiltonian system (let us recall that $\mathrm{H}$. Poincare has abundantly investigated the question of periodic solutions for finite-dimensional Hamiltonian systems; see [50]). Indeed if we set $p=u$ and $q=u_{t}$, then (1) becomes

$$
\frac{\partial}{\partial t}\left(\begin{array}{l}
p \\
q
\end{array}\right)=\left(\begin{array}{r}
H_{q} \\
-H_{p}
\end{array}\right)+\left(\begin{array}{l}
0 \\
f
\end{array}\right)
$$

where the Hamiltonian $H$ is defined on the space $H_{0}^{1}(0, \pi) \times L^{2}(0, \pi)$ by

$$
H(p, q)=\frac{1}{2} \int_{0}^{\pi}\left(p_{x}\right)^{2} d x+\int_{0}^{\pi} G(p) d x+\frac{1}{2} \int_{0}^{\pi} q^{2} d x
$$

and $G$ denotes a primitive of $g$.

We shall be concerned with two distinct questions.

Question 1. Existence of forced vibrations; that is, given $f(x, t)$ find at least one solution of (1).

Question 2. Existence of free vibrations (or “breathers"); that is, assume $f \equiv 0$ and find at least one nonzero solution of (1).

Received by the editors June 1, 1982.

1980 Mathematics Subject Classification. Primary 35 K60. 
In what follows we consider only the fixed period problem: $T$ is prescribed. In fact, we shall even assume systematically that $T / \pi$ is rational and sometimes we shall use for simplicity $T=2 \pi$. Such an assumption plays a technical ${ }^{1}$ but essential role in the proofs.

We point out that nothing is known for the fixed energy problem: that is, $f \equiv 0$ and $H\left(u, u_{t}\right)$-which is constant along solutions of $(1)$-is prescribed; even though much progress has been achieved in recent years on the fixed energy problem for finite-dimensional Hamiltonian systems. See e.g. A. Weinstein [6567], J. Moser [48], P. Rabinowitz [54, 55, 58] (the last reference is a survey article with many references), I. Ekeland-J. M. Lasry [36], A. Ambrosetti-G. Mancini [6], H. Hofer [40], and H. Berestycki-J. M. Lasry [14].

In $\S$ we state the main results. In §II, we discuss a dual variational principle which turns out to be extremely useful in solving (1). It is motivated by the dual variational formulation for finite-dimensional Hamiltonian systems discovered by F. Clarke and I. Ekeland (see [24, 25, 34]). In §III we present the proof of Theorem 1, which deals with forced vibrations. In §IV we sketch the proofs of Theorems 2.3 and 4 dealing with free vibrations.

Problem (1) has been approached in the sixties by purely analytical methods; we mention the works of O. Vejvoda [63], L. Cesari [21], J. Hale [38], P. Rabinowitz [51], H. Lovicarova [44], L. De Simon-G. Torelli [33] and the recent book of O. Vejvoda [64] which contains an extensive bibliography. In the seventies, the combination of analytical estimates with topological and variational tools (initiated by P. Rabinowitz in [52]) has shed a new light on Problem (1). We shall emphasize here this viewpoint.

I. The main results. Throughout this paper we shall assume that $g$ is a continuous nondecreasing function on $\mathbf{R}$, such that $g(0)=0$. Similar results hold when $g$ is nonincreasing. Howevex, very little is known if we drop the monotonicity assumptions (see, however, Remarks 5 and 10).

I.1. Forced vibrations. For simplicity we assume throughout subsection I.1 that $T=2 \pi$. We denote by $A$ the differential operator

$$
A u=u_{t t}-u_{x x}
$$

acting on functions which satisfy the Dirichlet boundary conditions in $x$ and which are $2 \pi$-periodic in $t$. We denote by $N(A)$ its null space so that $N(A)$ consists exactly of functions of the form

$$
\begin{array}{r}
N(A)=\{\varphi(x, t) ; \varphi(x, t)=p(t+x)-p(t-x) \\
\text { where } p \text { is any } 2 \pi \text {-periodic function }\} .
\end{array}
$$

THEOREM 1. Assume

$$
|g(u)| \leqslant \gamma|u|+C, \quad \forall u \in \mathbf{R}, \text { for some constants } \gamma<3 \text { and } C .
$$

\footnotetext{
${ }^{1}$ When $T / \pi$ is not rational we are led to unsolved difficulties related to small divisors.
} 
Assume $f(x, t) \in L^{\infty}$ admits a decomposition of the form

$$
f(x, t)=f^{*}(x, t)+f^{* *}(x, t)
$$

with

$$
\int_{0}^{\pi} \int_{0}^{2 \pi} f^{*} \varphi d x d t=0 \quad \text { for all } \varphi \in N(A)
$$

and

$$
g(-\infty)+\delta \leqslant f^{* *}(x, t) \leqslant g(+\infty)-\delta \text { for some } \delta>0, \text { for all } x, t .
$$

Then there exists a weak solution $u \in L^{\infty}$ of (1).

By a weak solution of (1) we mean a function $u \in L^{\infty}([0, \pi]) \times \mathbf{R}$ which is $T$-periodic and such that

$$
\int_{0}^{T} \int_{0}^{\pi} u\left(v_{t t}-v_{x x}\right) d x d t+\int_{0}^{T} \int_{0}^{\pi} g(u) v d x d t=\int_{0}^{T} \int_{0}^{\pi} f v d x d t
$$

for all $v \in C^{2}([0, \pi] \times \mathbf{R})$ satisfying the boundary and periodicity condition.

Theorem 1 is due to A. Bahri and myself [12]. If, in addition, $g$ satisfies $g( \pm \infty)= \pm \infty$, then any $f \in L^{\infty}$ admits a decomposition of the form (3), (4) (choose $f^{*}=0$ ). Therefore we obtain

Corollary 1. Assume that (2) holds and that $g( \pm \infty)= \pm \infty$. Then for every $f \in L^{\infty}$ there exists a weak solution $u \in L^{\infty}$ of (1).

Corollary 1 was first proved by Nirenberg and myself in [17] (some of the essential estimates devised in [17] will be presented in §III).

REMARK 1. The decomposability assumption on $f$ provides an "almost" necessary and sufficient condition for the solvability of (1). Indeed, if (1) has a solution then $f$ may be written as $f=f^{*}+f^{* *}$ where $f^{*}=A u$ and $f^{* *}=g(u)$. Thus, we find (3) and

$$
g(-\infty) \leqslant f^{* *}(x, t) \leqslant g(+\infty)
$$

In case $g$ is strictly increasing we may even conclude that (4) holds and then (3)-(4) is exactly a necessary and sufficient condition for the solvability of (1). Theorem 1 is related to the general "principle" which holds in many instances: the range of the sum is almost equal to the sum of the ranges, i.e. a nonlinear equation of the form $A u+B u=f$ has a solution provided $f$ admits a decomposition of the form $f=f^{*}+f^{* *}$ with $f^{*} \in R(A)$ and $f^{* *} \in R(B)$ (see $[18,29]$ ).

Suppose now that $-g(-\infty)=g(+\infty)=g_{\infty}$; using the Hahn-Banach theorem it is easy to see that (3)-(4) hold if and only if

$$
\left|\int_{0}^{\pi} \int_{0}^{2 \pi} f \varphi d x d t\right| \leqslant\left(g_{\infty}-\delta\right) \int_{0}^{\pi} \int_{0}^{2 \pi}|\varphi| d x d t
$$

for every $\varphi \in N(A)$ and for some $\delta>0$. Such a condition can be viewed as a nonlinear Fredholm alternative (of the same nature as the one used by Landesman-Lazer [42] in nonlinear equations at resonance; the main difference with 
their work is that the infinite-dimensional nullspace introduces further difficulties in our problem).

REMARK 2. Uniqueness can be proved (see [18, Corollary 1.6]) under the additional assumptions that $g$ is strictly increasing and that

$$
|g(u)-g(v)| \leqslant \gamma|u-v| \quad \text { for all } u, v \in \mathbf{R} \text { and for some } \gamma<3
$$

Nonuniqueness may otherwise occur (see Theorem 2 below).

REMARK 3. Smoothness. If $f, g$ are $C^{\infty}$ and furthermore $g$ is strictly increasing then any weak solution $u \in L^{\infty}$ of (1) belongs to $C^{\infty}$ (see [17, Theorem 2]).

REMARK 4. The restriction $\gamma<3$ in (2) is essential. Indeed, if $g(u)=3 u$ equation (1) does not have a solution for any $f$ : in fact (1) has a solution if and only if $f$ is orthogonal to $N(A+3 I) \neq\{0\}$ since -3 is an eigenvalue of $A$. Assumption (2) asserts that the nonlinear term $g$ is allowed to interact with the spectrum of $A$ only through 0 . A major difficulty arises here because 0 is an eigenvalue with infinite multiplicity. When $g$ does not interact with the spectrum of $A$ (the "nonresonant" case) or when $g$ "interacts" with one nonzero eigenvalue of $A$, Problem (1), becomes much easier (see J. Mawhin [46, 47], H. BrezisL. Nirenberg [18], G. Mancini [45], H. Amann-G. Mancini [1]). Nothing is known in case $g$ grows at infinity faster than linear, for example, $g(u)=u^{3}$. In view of recent results ${ }^{2}$ of A. Bahri [8], A. Bahri-H. Berestycki [9-11], M. Struwe [60] and P. Rabinowitz [59] it seems reasonable to conjecture that when $g(u)=u^{3}$, Problem (1) possesses a solution-even infinitely many solutions-for every $f$ (or at least for a dense set of $f$ 's).

REMARK 5. We have assumed that $g$ is monotone. This assumption plays an essential role in the proofs which involve an interplay of monotonicity and compactness devices. Such a combination has been extensively used in the past since the pioneering works of F. Browder [19] and J. Leray-J. L. Lions [43] dealing with nonlinear elliptic (and parabolic) problems. In that case the top order differential operator is monotone (i.e., elliptic) and there is much freedom on the lower order terms - which are handled by compactness. For Problem (1) the situation is reversed: the top order differential operator $A$ is not monotone, but $A^{-1}$ (off its nullspace) is compact; the monotonicity of the lower order term $g(u)$ is used in a crucial manner (because $N(A)$ is infinite dimensional and no compactness is available on $N(A)$ ).

Let us examine a simple example of a nonmonotone $g$ : consider Problem (1) with $g(u)=u+36 \sin u$. It is not known whether (1) possesses a solution for every $f$ (even though the solutions of (1) are a priori bounded in $L^{\infty}$, but such an estimate is too weak for proving existence). Recently, H. Hofer [39] has proved that (1) possesses a solution for a dense set of $f$ 's (see also an earlier work by

\footnotetext{
${ }^{2}$ Dealing with elliptic equations and finite-dimensional Hamiltonian systems.
} 
M. Willem [68]). On the other hand, J. M. Coron [32] has proved that (1) possesses a solution for every $f$ satisfying the additional symmetry assumption:

$$
f(\pi-x, \pi+t)=f(x, t)
$$

for all $x \in[0, \pi]$, and $t \in \mathbf{R}$.

I.2. Free vibrations. We assume that $f \equiv 0$. By a nontrivial solution of (1) we mean a function $u \in L^{\infty}$ satisfying (1) in the weak sense and such that $g(u) \neq 0$ on a set $(x, t)$ of positive measure (in particular $u \neq 0$ on that set).

The main results are the following:

TheOREM 2. Assume (2) and

$$
\liminf _{u \rightarrow 0} \frac{g(u)}{u}>3
$$

Then there exists a nontrivial $2 \pi$-periodic solution of (1).

TheOREM 3. Assume $g \neq 0$ and

$$
\lim _{|u| \rightarrow \infty} \frac{g(u)}{u}=0 .
$$

Then there exists some $T_{0}$ such that for each $T>T_{0}$ which is a rational multiple of $\pi$, equation (1) admits a nontrivial T-periodic solution.

THEOREM 4. Assume

$$
\begin{gathered}
\qquad \lim _{|u| \rightarrow \infty} \frac{g(u)}{u}=\infty \\
\left\{\begin{array}{l}
\text { there are constants } \alpha>0 \text { and } C \text { such that } \\
\frac{1}{2} u g(u)-G(u) \geqslant \alpha|g(u)|-C \text { for all } u \in \mathbf{R}, \\
\text { where } G(u)=\int_{0}^{u} g(t) d t .
\end{array}\right.
\end{gathered}
$$

Then for each $T$ which is a rational multiple of $\pi$, equation (1) admits a nontrivial T-periodic solution.

Theorem 2 is due to J. M. Coron [29]; Theorem 3 is due to J. M. Coron and myself [15]. Theorem 4 is due to P. Rabinowitz [53] under slightly more restrictive assumptions on $g$. As stated here, Theorem 4 is due to J. M. Coron-L. Nirenberg and myself [16]; the proof in [16] is simpler than the original proof of P. Rabinowitz. I shall sketch it in §IV.

REMARK 6. It is tempting to "visualize" Theorems 2,3 , and 4 as follows. In Theorem 2 replace $A u$ by $-3 u$ ( -3 plays a special role as the first negative eigenvalue of $A$ ). Under the assumptions of Theorem 2 the scalar equation $-3 u+g(u)=0$ admits a nontrivial solution. More generally, denote by $\lambda_{-1}(T)$ the first negative eigenvalue of $A$ acting in $T$-periodic functions. As we shall see (in $\S \mathrm{IV}),\left|\lambda_{-1}(T)\right| \rightarrow 0$ as $T \rightarrow \infty$. Under the assumptions of Theorem 3 the scalar equation $\lambda_{-1}(T) u+g(u)=0$ admits a nontrivial solution if $T$ is large 
enough. Finally, in Theorem 4 we may as well look for a $T / n$-periodic solution of (1) ( $n$ integer) instead of a $T$-periodic solution. Since $\lambda_{-1}(T / n) \rightarrow-\infty$ as $n \rightarrow \infty$, it is clear, with assumption (7), that the scalar equation $\lambda_{-1}(T / n) u+g(u)=0$ has a nontrivial solution for $n$ large enough.

REMARK 7. Under the assumptions of Theorem 3, and in addition if $\lim _{u \rightarrow 0}(g(u) / u)=0$, then J. M. Coron [30] proves that there exist at least two nontrivial solutions of (1) for $T$ large enough. Again, the scalar analogy is suggestive.

REMARK 8. Other existence and multiplicity results have been obtained by $\mathrm{H}$. Amann-E. Zehnder [2, 3], K. C. Chang [22], K. C. Chang-S. P. Wu-S. Li [23], in case $g(u)$ has a linear behavior as $|u| \rightarrow \infty$. Roughly speaking, their results assert that if the interval joining $g^{\prime}(0)$ to $g^{\prime}(\infty)$ crosses $m$ eigenvalues of $-A$, then (under some extra technical assumptions) Problem (1) possesses at least $m$ nontrivial solutions; the proofs rely on the use of Morse and Lusternik-Schnirelmann arguments.

REMARK 9. P. Rabinowitz has obtained further results related to Theorem 4. In [56] he investigates the existence of subharmonic solutions of (1) (that is distinct solutions of period $n T$ ). In [57] he studies the case where $g$ is monotone decreasing (instead of increasing).

REMARK 10. J. M. Coron [32] has recently succeeded in removing the monotonicity assumption (on $g$ ) in most of the previous results (at the expense of some extra technical assumptions). When $g$ is not monotone the major difficulty arises from the infinite-dimensional null space $N(A)$ (see Remark 5). In order to overcome this difficulty Coron looks for a nontrivial solution of (1) within a restricted class $H_{1}$ of functions satisfying some symmetry properties and such that

(i) $N(A) \cap H_{1}=\{0\}$,

(ii) $H_{1}$ is stable under $A$ and $g$.

Consider (for example) $g(u)=\sin u$; Coron proves that given any integer $m$, Problem (1) has at least $m$ distinct solutions provided $T$ is large enough. Unfortunately, this device does not apply when $g(x, t, u)$ depends also on $(x, t)$ - except if $g$ satisfies some special symmetry properties.

REMARK 11. It is of great interest (see [20]) to study the existence of nontrivial solutions for a problem similar to (1), when $x$ varies on $\mathbf{R}$ instead of the bounded interval $[0, \pi]$; that is

$$
\begin{cases}u_{t t}-u_{x x}+g(u)=0, & -\infty<x<+\infty, t \in \mathbf{R} \\ u(x, t) \rightarrow 0, & \text { as }|x| \rightarrow \infty, t \in \mathbf{R} \\ u(x, t+T)=u(x, t), & -\infty<x<+\infty, t \in \mathbf{R}\end{cases}
$$

When $g(u)=\sin u$ an explicit solution is known to exist for every period $T>2 \pi$ (see e.g. G. Lamb [41]):

$$
u(x, t)=4 \arctan \left[\frac{\varepsilon \sin (2 \pi t / T)}{\cosh (2 \pi \varepsilon x / T)}\right] \text { where } \varepsilon=\left[\left(\frac{T}{2 \pi}\right)^{2}-1\right]^{1 / 2}
$$


It has been proved by J. M. Coron [31], for a general function $g$, that nontrivial $T$-periodic solutions of $\left(1^{\prime}\right)$ can exist only when

$$
g^{\prime}(0)>(2 \pi / T)^{2}
$$

In particular, if $g(u)=\sin u$, there is no $T$-periodic solution when $T<2 \pi$ (this is consistent with the above formula). When $g(u)=-\sin u$ or $g(u)= \pm u^{3}$ then $u \equiv 0$ is the only solution of $\left(1^{\prime}\right)$ (for any period $T$ ). It seems that the class of nonlinearities $g(u)$ for which $\left(1^{\prime}\right)$ has a solution is very limited (perhaps only multiples of $\sin u$ !).

II. A duality principle. Recently, F. Clarke and I. Ekeland have discovered an interesting dual variational formulation ${ }^{3}$ for Hamiltonian systems associated with a convex Hamiltonian $H$ (see [24, 25, 35]). Such a duality principle has turned out to be extremely useful for various purposes (see [4-6], [26-28], [35-37]). While analyzing this principle and trying to adapt it to Problem (1) we were led to the following abstract scheme which fits both Hamiltonian systems (with convex $H$ ) and Problem (1) (with monotone $g$ ):

Let $H$ be a Hilbert space and let $A: D(A) \subset H \rightarrow H$ be an unbounded linear operator such that $A^{*}=A$ and $R(A)$ is closed. Thus $H$ admits an orthogonal decomposition $H=R(A) \oplus N(A)$; for each $u \in H$ we write $u=u_{1}+u_{2}$ with $u_{1} \in R(A), u_{2} \in N(A) . A^{-1}$ is a well-defined bounded operator from $R(A)$ into $R(A)$.

Let $J$ be a $C^{1}$ convex function on $H$ and set $B=\nabla J$. Assume $B$ is one-to-one and onto. Consider the equation

$$
A u+B u=f .
$$

Equation (9) has a natural variational structure: the solutions of (9) correspond to the critical points of the functional

$$
\Phi(u)=\frac{1}{2}(A u, u)+J(u)-(f, u)
$$

on $D(A)$.

We introduce the new function

$$
\dot{v}=B u-f
$$

Solving (9) is clearly equivalent to finding $v$ such that

$$
\left\{\begin{array}{l}
v \in R(A), \\
A^{-1} v+B^{-1}(v+f) \in N(A) .
\end{array}\right.
$$

\footnotetext{
${ }^{3}$ Prior to their works, J. F. Toland $[\mathbf{6 1}, 6 \mathbf{6 2}]$ had introduced a somewhat related idea in the study of variational problems involving a difference of two convex functions.
} 
Problem (1) has also a variational structure. Namely the solutions of (2) correspond to the critical points of the functional

$$
\left\{\begin{array}{l}
\Psi(v)=\frac{1}{2}\left(A^{-1} v, v\right)+J^{*}(v+f) \\
\text { subject to the constraint } v \in R(A)
\end{array}\right.
$$

where $J^{*}$ denotes the conjugate convex function (i.e., Legendre transform) of $J$.

We say that (13) is the dual variational formulation of Problem (9). It is much easier in practice to find critical points of $\Psi$ (on $R(A)$ ) than critical points of $\Phi$. The reasons are the following:

(a) In general, $A$ has an infinite sequence of eigenvalues going from $-\infty$ to $+\infty$, so that $\Phi$ is unbounded from above and below. Critical points of $\Phi$ can never be obtained by a simple minimization (or maximization). On the other hand, in general, $A^{-1}$ is compact, and so $\Psi$ satisfies a condition of the Palais-Smale type. Therefore one can make use of classical techniques in order to find critical points of $\Psi$. It may even happen in some cases that $J^{*}(v+f)$ "dominates" the negative part of $\frac{1}{2}\left(A^{-1} v, v\right)$; then $\Psi$ is coercive and a critical point may be obtained by simply minimizing $\Psi$ on $R(A)$. Note that Rabinowitz [53] works with $\Phi$ rather than $\Psi$; this explains why his argument is more intricate.

(b) Another reason is that, in problem (9), the component of $u$ in $N(A)$ is difficult to estimate. It disappears from problem (12) - or rather it appears in (12) as a "harmless" Lagrange multiplier (a comparable device is used in solving the Navier-Stokes equation when the pressure term is turned into a Lagrange multiplier by projecting the equation on divergence free vector fields).

When applying this scheme to problem (1) we choose $H=L^{2}((0, \pi) \times(0, T))$,

$$
\left\{\begin{array}{l}
A u=u_{t t}-u_{x x}, \quad \text { under appropriate boundary and periodicity conditions, } \\
B u=g(u) .
\end{array}\right.
$$

For the study of the finite-dimensional Hamiltonian system

$$
\left\{\begin{aligned}
-\frac{d p}{d t}+H_{q} & =f_{1}, \\
\frac{d q}{d t}+H_{p} & =f_{2}
\end{aligned}\right.
$$

we choose $H=L^{2}(0, T) \times L^{2}(0, T), u=\left(\begin{array}{c}p \\ q\end{array}\right)$,

$$
\begin{aligned}
& A u=\left(\begin{array}{r}
\frac{d q}{d t} \\
-\frac{d p}{d t}
\end{array}\right)=\left(\begin{array}{cc}
0 & I \\
-I & 0
\end{array}\right)\left(\frac{d u}{d t}\right) \text { under periodic conditions and } \\
& B u=\left(\begin{array}{l}
H_{p} \\
H_{q}
\end{array}\right)=\nabla H(u) .
\end{aligned}
$$


III. Proof of Theorem 1. The duality principle of $\S$ II applies provided $B$ is one-to-one and onto. This is not always the case under the assumptions of Theorem 1. Therefore we consider first the perturbed equation

$$
A u_{\varepsilon}+g\left(u_{\varepsilon}\right)+\varepsilon u_{\varepsilon}=f .
$$

The proof of Theorem 1 is divided into 4 steps.

Step 1. Existence of a solution $u_{\varepsilon}$ of (14).

Step 2. "Soft" estimates for $u_{\varepsilon}$ :

$$
\left\|u_{\varepsilon}\right\|_{L^{1}} \leqslant C, \quad\left\|A u_{\varepsilon}\right\|_{L^{2}} \leqslant C, \quad\left\|g\left(u_{\varepsilon}\right)\right\|_{L^{2}} \leqslant C .
$$

Step 3. A further estimate, $\left\|u_{\varepsilon}\right\|_{L^{\infty}} \leqslant C$.

Step 4. Passage to the limit as $\varepsilon \rightarrow 0$.

Step 1 . Set $\Omega=(0, \pi) \times(0,2 \pi)$. We recall some properties of $A$ in $L^{2}(\Omega)$ (see [17] and the references quoted therein):

$$
A^{*}=A \text {, }
$$

$$
R(A) \text { is closed in } L^{2} \text {. }
$$

Set $K=A^{-1}$ defined from $R(A)$ into $R(A)$. The eigenvalues of $A$ are $\left\{j^{2}-k^{2}\right.$; $j=1,2,3, \ldots, k=0,1,2, \ldots\}$. The corresponding eigenfunctions are $\sin j x \sin k t$ and $\sin j x \cos k t$. In particular -3 is the first negative eigenvalue of $A$ and so

$$
(A u, u) \geqslant-\frac{1}{3}\|A u\|_{L^{2}}^{2} \quad \text { for all } u \in D(A)
$$

or equivalently

$$
(K f, f) \geqslant-\frac{1}{3}\|f\|_{L^{2}}^{2} \quad \text { for all } f \in R(A) .
$$

$K$ is a compact operator in $L^{2}$ and in addition

$$
\begin{array}{cc}
\|K f\|_{L^{\infty}} \leqslant C\|f\|_{L^{1}} & \text { for all } f \in R(A), \\
\|K f\|_{H^{1}} \leqslant C\|f\|_{L^{2}} & \text { for all } f \in R(A) .
\end{array}
$$

We shall prove that for $\varepsilon<3-\gamma$, equation (14) has a solution $u_{\varepsilon}$. Indeed set $g_{\varepsilon}(u)=g(u)+\varepsilon u$ and let $h_{\varepsilon}$ be the inverse function of $g_{\varepsilon}$. Set $H_{\varepsilon}(t)=\int_{0}^{t} h_{\varepsilon}(s) d s$.

In view of the duality principle of $\S I$ applied to $B u=g(u)+\varepsilon u$, equation (14) has a solution provided we can find a critical point of

$$
\Psi_{\varepsilon}(v)=\frac{1}{2} \int_{\Omega} K v \cdot v+\int_{\Omega} H_{\varepsilon}(v+f)
$$

subject to the constraint $v \in R(A)$.

We need the following:

Lemma 1. There are constants $\alpha>0$ and $C$ such that

$$
\Psi_{\varepsilon}(v) \geqslant \alpha\|v\|_{L^{2}}^{2}-C \text { for all } v \in R(A) .
$$


Proof. We have

$$
\left|g_{\varepsilon}(u)\right| \leqslant(\gamma+\varepsilon)|u|+C \quad \text { for all } u \in \mathbf{R}
$$

i.e.,

$$
|v| \leqslant(\gamma+\varepsilon)\left|h_{\varepsilon}(v)\right|+C \quad \text { for all } v \in \mathbf{R}
$$

and so

$$
H_{\varepsilon}(v) \geqslant \frac{1}{2(\gamma+\varepsilon)}|v|^{2}-C|v| \quad \text { for all } v \in \mathbf{R} .
$$

We easily derive (20) from (17') and (21).

It follows from Lemma 1 that $\Psi_{\varepsilon}$ achieves its minimum on $R(A)$ (note that $\Psi_{\varepsilon}$ is lower semicontinuous for the weak topology).

Step 2. For any solution $u_{\varepsilon}$ of (14) we have

$$
\left\|u_{\varepsilon}\right\|_{L^{1}} \leqslant C, \quad\left\|A u_{\varepsilon}\right\|_{L^{2}} \leqslant C, \quad\left\|g\left(u_{\varepsilon}\right)\right\|_{L^{2}} \leqslant C .
$$

In what follows we denote by $C$ various constants independent of $\varepsilon$. We deduce from (2) and (4) that

$$
\begin{gathered}
\left(g(u)-f^{* *}(x, t)\right) u \geqslant \frac{\delta}{2}|u|-C, \quad \text { for all } u \in \mathbf{R} \text { and }(x, t) \in \Omega, \\
\left(g(u)-f^{* *}(x, t)\right) u \geqslant \frac{1}{\gamma}|g(u)|^{2}-C|g(u)|-C \\
\text { for all } u \in \mathbf{R},(x, t) \in \Omega .
\end{gathered}
$$

Choosing $u=u_{\varepsilon}$ in (24) and using (17) we find

$$
\varepsilon\left\|u_{\varepsilon}\right\|_{L^{2}}^{2}+\frac{1}{\gamma}\left\|g\left(u_{\varepsilon}\right)\right\|_{L^{2}}^{2} \leqslant \frac{1}{3}\left\|A u_{\varepsilon}\right\|_{L^{2}}^{2}=C\left\|A u_{\varepsilon}\right\|_{L^{2}}+C\left\|g\left(u_{\varepsilon}\right)\right\|_{L^{2}}+C .
$$

Replacing $g\left(u_{\varepsilon}\right)$ by $\left(f-A u_{\varepsilon}-\varepsilon u_{\varepsilon}\right)$ in (25) we conclude that

$$
\left\|A u_{\varepsilon}\right\|_{L^{2}} \leqslant C \text { and }\left\|g\left(u_{\varepsilon}\right)\right\|_{L^{2}} \leqslant C \text {. }
$$

Finally we choose $u=u_{\varepsilon}$ in (23) and we obtain $\left\|u_{\varepsilon}\right\|_{L^{1}} \leqslant C$.

Step 3. We shall prove that

$$
\left\|u_{\varepsilon}\right\|_{L^{\infty}} \leqslant C .
$$

We write $u_{\varepsilon}=u_{1 \varepsilon}+u_{2 \varepsilon}$ with $u_{1 \varepsilon}+u_{2 \varepsilon}$ with $u_{1 \varepsilon} \in R(A), u_{2 \varepsilon} \in N(A)$. By (22) and (18) we already know that

$$
\left\|u_{1 \varepsilon}\right\|_{L^{\infty}} \leqslant C .
$$

Therefore it suffices to prove that $\left\|u_{2 \varepsilon}\right\|_{L^{\infty}} \leqslant C$.

Since $u_{2 \varepsilon} \in N(A), u_{2 \varepsilon}$ has the form

$$
u_{2 \varepsilon}(x, t)=p_{\varepsilon}(t+x)-p_{\varepsilon}(t-x)
$$

where $p_{\varepsilon}$ is a $2 \pi$-periodic function with $\int_{0}^{2 \pi} p_{\varepsilon}=0$, given by

$$
p_{\varepsilon}(t)=\frac{1}{2 \pi} \int_{0}^{\pi}\left[u_{2 \varepsilon}(x, t-x)-u_{2 \varepsilon}(x, t+x)\right] d x
$$


so that, by (22) we obtain

$$
\left\|p_{\varepsilon}\right\|_{L^{1}} \leqslant C
$$

For simplicity we write $p=p_{\varepsilon}$.

On the other hand we observe that a function $\psi \in L^{2}$ belongs to $N(A)^{\perp}$ if and only if

$$
\int_{0}^{\pi}[\psi(x, t-x)-\psi(x, t+x)] d x=0 \text { for a.e. } t \text {. }
$$

Indeed $\psi \in N(A)^{\perp}$ if and only if $\int_{\Omega} \psi(x, t)[q(t+x)-q(t-x)] d x d t=0$ for every $2 \pi$-periodic function $q$. Since $g\left(u_{\varepsilon}\right)+u_{2 \varepsilon}-f^{* *} \in N(A)^{\perp}$ we have

$$
\left\{\begin{array}{l}
\varepsilon p(t)+\frac{1}{2 \pi} \int_{0}^{\pi}\left[g\left(u_{\varepsilon}(x, t-x)\right)-g\left(u_{\varepsilon}(x, t+x)\right)\right] d x \\
=\frac{1}{2 \pi} \int_{0}^{\pi}\left[f^{* *}(x, t-x)-f^{* *}(x, t+x)\right] d x \text { for a.e. } t .
\end{array}\right.
$$

From (27) we deduce that

$u_{\varepsilon}(x, t-x) \geqslant-C+p(t)-p(t-2 x), \quad u_{\varepsilon}(x, t+x) \leqslant C+p(t+2 x)-p(t)$ and by (4) we have

$$
f^{* *}(x, t-x) \leqslant g(+\infty)-\delta, \quad f^{* *}(x, t+x) \geqslant g(-\infty)+\delta .
$$

We derive from (29) that

$$
\begin{gathered}
\varepsilon p(t)+\frac{1}{2 \pi} \int_{0}^{\pi}[g(-C+p(t)-p(t-2 x))-g(C+p(t+2 x)-p(t))] d x \\
\leqslant \frac{1}{2}[g(+\infty)-g(-\infty)]-\delta \text { for a.e. } t .
\end{gathered}
$$

Finally we set $\tilde{g}(u)=\frac{1}{2}(g(u)-g(-u))$ and we find

$$
\varepsilon p(t)+\frac{1}{2 \pi} \int_{0}^{2 \pi} \tilde{g}(-C+p(t)-p(s)) d x \leqslant \tilde{g}(+\infty)-\delta \text { for a.e. } t \text {. }
$$

Let $M=$ ess $\sup _{t \in(0,2 \pi)} p(t) \geqslant 0$; our objective is to obtain a bound for $M$ independent of $\varepsilon$.

By (30) we have

$$
\frac{1}{2 \pi} \int_{0}^{2 \pi} \tilde{g}(-C+M-p(s)) d x \leqslant \tilde{g}(+\infty)-\delta .
$$

On the other hand let

$$
\Sigma=\{s \in(0,2 \pi) ; p(s) \geqslant M / 2\} .
$$

By (28) we know that $M m(\Sigma) / 2 \leqslant C$. Also, we have

$$
\int_{0}^{2 \pi} \tilde{g}(-C+M-p(s)) d s=\int_{\Sigma} \int_{c_{\Sigma}} \geqslant m(\Sigma) \tilde{g}(-C)+m\left({ }^{c} \Sigma\right) \tilde{g}\left(-C+\frac{M}{2}\right) .
$$

Suppose $M \geqslant 2 C$; we deduce from (31) that

$$
\frac{1}{2 \pi}\left(2 \pi-\frac{2 C}{M}\right) \tilde{g}\left(\frac{M}{2}-C\right) \leqslant \tilde{g}(C) \frac{2 C}{M}+\tilde{g}(+\infty)-\delta
$$


-which prevents $M$ from going to infinity as $\varepsilon \rightarrow 0$. Therefore $p \leqslant C$; similarly $p \geqslant-C$ and we conclude that $\|p\|_{L^{\infty}} \leqslant C$.

Step 4. Passage to the limit.

Using the estimates of Steps 2 and 3 we may extract a sequence $\varepsilon_{n} \rightarrow 0$ such that

$$
\begin{array}{ll}
u_{\varepsilon_{n}} \rightarrow u & \text { weakly in } L^{2}, \\
A u_{\varepsilon_{n}} \rightarrow A u & \text { weakly in } L^{2}, \\
u_{1 \varepsilon_{n}} \rightarrow u_{1} & \text { strongly in } L^{2} .
\end{array}
$$

We need Minty's device in order to pass to the limit in the nonlinear term $g\left(u_{\varepsilon}\right)$.

We have

$$
\left(g\left(u_{\varepsilon}\right)-g(\varphi), u_{\varepsilon}-\varphi\right) \geqslant 0 \text { for all } \varphi \in L^{2},
$$

i.e.,

$$
\left(f-A u_{\varepsilon}-\varepsilon u_{\varepsilon}-g(\varphi), u_{\varepsilon}-\varphi\right) \geqslant 0 .
$$

Note that $\left(A u_{\varepsilon}, u_{\varepsilon}\right)=\left(A u_{\varepsilon}, u_{1 \varepsilon}\right)$ and so $\left(A u_{\varepsilon_{n}}, u_{\varepsilon_{n}}\right) \rightarrow(A u, u)$. Passing to the limit in (32) we find

$$
(f-A u-g(\varphi), u-\varphi) \geqslant 0 \text { for all } \varphi \in L^{2} .
$$

We conclude in the usual manner, choosing $\varphi=u+t \psi$, that $u$ satisfies $A u+g(u)=f$.

\section{Proofs of Theorems 2.3 and 4.}

Proof of Theorem 2. We start with the perturbed problem

$$
A u_{\varepsilon}+g\left(u_{\varepsilon}\right)+\varepsilon u_{\varepsilon}=0 .
$$

As we know, the solutions of (33) correspond to critical point of

$$
\Psi_{\varepsilon}(v)=\frac{1}{2} \int_{\Omega} K v \cdot v+\int_{\Omega} H_{\varepsilon}(v)
$$

on $R(A)$-through the relation $v=g(u)+\varepsilon u$. Clearly 0 is a critical point of $\Psi_{\varepsilon}$; we shall obtain a nontrivial critical point and then pass to the limit.

We already know (see the proof of Theorem 1), that $\operatorname{Inf}_{R(A)} \psi_{\varepsilon}$ is achieved, say at $v_{\varepsilon} \in R(A)$.

We end the following

LEMMA 2. $\operatorname{Inf}_{R(A)} \psi_{\varepsilon} \leqslant-m$, where $m>0$ is a constant independent of $\varepsilon$.

Proof of Lemma 2. From (5) we deduce that $|g(u)| \geqslant \theta|u|$ for some constant $\theta>3$ and for $u$ near 0 and so $\left|g_{\varepsilon}(u)\right| \geqslant \theta|u|$ for $u$ near 0 . Hence $|v| \geqslant \theta\left|h_{\varepsilon}(v)\right|$ for $v$ near 0 and $H_{\varepsilon}(v) \leqslant|v|^{2} / 2 \theta$ for $v$ near 0 . Let $v_{0}=\sin x \sin 2 t$ be an eigenfunction of $K$ corresponding to the eigenvalue $-1 / 3$. We have

$$
\psi_{\varepsilon}\left(t v_{0}\right)=-\frac{t^{2}}{6}\left\|v_{0}\right\|_{L^{2}}^{2}+\int_{\Omega} H_{\varepsilon}\left(t v_{0}\right)
$$


and for $t$ near 0

$$
\psi_{\varepsilon}\left(t v_{0}\right) \leqslant\left(-\frac{1}{6}+\frac{1}{2 \theta}\right) t^{2}\left\|v_{0}\right\|_{L^{2}}^{2}
$$

The conclusion of Lemma 2 follows directly.

Proof of TheOrem 2 concluded. From Lemma 2 we obtain a nontrivial solution $u_{\varepsilon}$ of (33) related to $v_{\varepsilon}$ by the relation $g\left(u_{\varepsilon}\right)+\varepsilon u_{\varepsilon}=v_{\varepsilon}$. We know (see the proof of Theorem 1) that $\left\|u_{\varepsilon}\right\|_{L^{\infty}} \leqslant C$. Therefore we may extract a sequence $\varepsilon_{n} \rightarrow 0$ such that $u_{\varepsilon_{n}} \rightarrow u, A u_{\varepsilon_{n}} \rightarrow A u, g\left(u_{\varepsilon_{n}}\right) \rightarrow g(u), v_{\varepsilon_{n}} \rightarrow g(u)$ weakly in $L^{2}$.

By Lemma 2 we have $\frac{1}{2} \int_{\Omega} K v_{\varepsilon} \cdot v_{\varepsilon} \leqslant-m$. Hence, at the limit $\frac{1}{2} \int_{\Omega} K g(u) \cdot g(u) \leqslant$ $-m$, and $u$ is a nontrivial solution of $A u+g(u)=0$.

SKeTCH OF THE PROOF of TheOREM 3. We consider now the problem of finding $T$-periodic solutions. Since $T$ is a rational multiple of $\pi$ we may write $T=2 \pi b / a$ where $a$ and $b$ are coprime integers.

Set $\Omega=(0, \pi) \times(0, T)$; we consider in $L^{2}(\Omega)$ the operator $A u=u_{t t}-u_{x x}$ acting on functions satisfying the boundary and $T$-periodicity conditions. As in $\S$ III we have (15), (16), (18) and (19). There are two differences with the case $T=2 \pi$. Here,

$$
N(A)=\{p(t+x)-p(t-x), \text { where } p \text { has period } 2 \pi / a=T / b\} ;
$$

the eigenvalues of $A$ consist of

$$
\left\{j^{2}-(2 \pi k / T)^{2} ; j=1,2,3, \ldots, k=0,1,2, \ldots\right\}
$$

and they correspond to eigenfunctions $\sin j x \sin (2 \pi k t / T), \sin x \cos (2 \pi k t / T)$. Let $\lambda_{-1}(T)$ be the first negative eigenvalue of $A$. Instead of (17) we have

$$
(A u, u) \geqslant \frac{1}{\left|\lambda_{-1}(T)\right|}\|A u\|_{L^{2}} \quad \text { for all } u \in D(A) .
$$

Note that $\lambda_{-1}(T) \rightarrow 0$ as $T \rightarrow \infty$. Indeed let $\mu=j^{2}-(2 \pi k / T)^{2}$ with $j=1$ and $k=[T / 2 \pi]+1$.

We have $1-(1+2 \pi / T)^{2} \leqslant \mu<0$ and so $\left|\lambda_{-1}(T)\right| \leqslant|\mu| \leqslant 4 \pi(1+\pi / T) / T$. We use the same approach as in the proof of Theorem 2 , i.e., minimize $\Psi_{\varepsilon}$ on $R(A)$. We shall need the following lemma.

LEMMA 3. There exists $T_{0}$ such that if $T>T_{0}$ and $T$ is a rational multiple of $\pi$, then

$$
\operatorname{Inf}_{R(A)} \Psi_{\varepsilon} \leqslant-1 \quad(\varepsilon>0) .
$$

Proof of Lemma 3. Since $g(u) \neq 0$ we may assume for example that

$$
g(u) \geqslant \delta>0 \text { for } u \geqslant R .
$$

Therefore

$$
h_{\varepsilon}(v) \leqslant R \text { for } 0 \leqslant v \leqslant \delta
$$

and

$$
H_{\varepsilon}(v) \leqslant C \quad \text { for } 0 \leqslant v \leqslant \delta .
$$


Let $v_{0}=\sin j x \sin (2 \pi k t / T)$ be an eigenfunction of $A$ corresponding to the eigenvalue $\lambda_{-1}(T)$. Let $v=\delta\left(1+v_{0}\right) / 2$. We have $K(1)=-x(x-\pi) / 2$ and $K v_{0}=v_{0} / \lambda_{-1}(T)$. Thus

$$
(K v, v)=\frac{\delta^{2}}{4} \iint\left[-\frac{x}{2}(x-\pi)+\frac{1}{\lambda_{-1}(T)} v_{0}^{2}\right] d x d t=\frac{1}{16} \delta^{2} \pi\left[\frac{\pi^{2}}{3}+\frac{1}{\lambda_{-1}(T)}\right]
$$

and

$$
\Psi_{\varepsilon}(v) \leqslant \frac{T}{32} \delta^{2} \pi\left[\frac{\pi^{2}}{3}+\frac{1}{\lambda_{-1}(T)}\right]+C T \rightarrow-\infty \quad \text { as } T \rightarrow+\infty .
$$

Hence $\operatorname{Inf}_{R(A)} \Psi_{\varepsilon} \leqslant-1$ provided $T \geqslant T_{0}$ for some large $T_{0}$.

In what follows we fix $T \geqslant T_{0}$.

Proof OF THEOREM 3 CONCLUded. By minimizing $\Psi_{\varepsilon}$ on $R(A)$ we obtain-as in the proof of Theorem 2-a solution of the equation

$$
A u_{\varepsilon}+g\left(u_{\varepsilon}\right)+\varepsilon u_{\varepsilon}=0
$$

such that $v_{\varepsilon}=g\left(u_{\varepsilon}\right)+\varepsilon u_{\varepsilon}$ satisfies

$$
\frac{1}{2} \int K v_{\varepsilon} \cdot v_{\varepsilon} \leqslant-1 .
$$

[Note that Lemma 1 holds since, by (6), we have $|g(u)| \leqslant \gamma|u|+C$, for all $u$, with $\gamma<\left|\lambda_{-1}(T)\right|$.]

Using the same technique as in the proof of Theorem 1 we show that $\left\|u_{\varepsilon}\right\|_{L^{\infty}} \leqslant C$ and we pass to the limit as $\varepsilon \rightarrow 0$. (The only difference is that, instead of (23) we have

$$
g(u) \cdot u \geqslant \delta\left|u^{+}\right|-C \text { for all } u \in \mathbf{R}
$$

which leads to $\int u_{\varepsilon}^{+} \leqslant C$ and then $\int\left|u_{\varepsilon}\right|=2 \int u_{\varepsilon}^{+}-\int u_{\varepsilon}=2 \int u_{\varepsilon}^{+}-\int u_{\varepsilon} \leqslant C$ since $\int u_{2 \varepsilon}=0$.)

SKETCH OF THE PROOF OF THEOREM 4. For simplicity I shall describe the proof in the special case where $g(u)=|u|^{m-2} u, m>2$ and $T=2 \pi$. In the general case the proof is technically more complicated, but along the same lines (see [16] for the details). Set $v=g(u)=|u|^{m-2} u$. Using the dual formulation we have to find a nontrivial critical point for the functional

$$
\Psi(v)=\frac{1}{2} \int K v \cdot v+\frac{1}{m^{\prime}} \int|v|^{m^{\prime}}
$$

subject to the constraint $v \in E$, where $1 / m+1 / m^{\prime}=1$ and $E=\left\{v \in L^{m^{\prime}}(\Omega)\right.$; $\int v \varphi=0$ for all $\left.\varphi \in N(A)\right\}$. Note that, by (18), $K$ maps $E$ into $L^{\infty}$ and that $K$ is compact from $E$ into $L^{m}(\Omega)$. Clearly $\Psi$ is $C^{1}$ on $E$; in fact

$$
\left\langle\Psi^{\prime}(v), \zeta\right\rangle_{E^{*}, E}=\int K v \cdot \zeta+\int|v|^{m^{\prime}-2} v \zeta \text { for all } v, \zeta \in E .
$$

Using the Hahn-Banach theorem we may write

$$
K v+|v|^{m^{\prime}-2} v=w+\varphi
$$


with $w \in L^{m},\|w\|_{L^{m}}=\left\|\Psi^{\prime}(v)\right\|_{E^{*}}$ and $\varphi \in N(A) \cap L^{m}$. Here, $\operatorname{Inf}_{E} \Psi=-\infty$ and $\operatorname{Sup}_{E} \Psi=+\infty$; thus we can no longer find a critical point by minimization of maximization. We shall, instead, rely on the following geometrical result due to A. Ambrosetti-P. Rabinowitz [7]. (See also [13] and [49].)

Lemma (Mountain Pass Lemma). Assume $\Psi$ is a $C^{1}$ function on a Banach space $E$ and satisfies the Palais-Smale condition, i.e.

$$
\left\{\begin{array}{l}
\text { whenever a sequence }\left\{v_{j}\right\} \text { in } E \text { satisfies }\left|\Psi\left(v_{j}\right)\right| \leqslant C \\
\text { and } \Psi^{\prime}\left(v_{j}\right) \rightarrow 0 \text { in } E^{*} \text { there exists a subsequence of } v \\
\text { which converges in } E .
\end{array}\right.
$$

Assume also

$$
\begin{gathered}
\left\{\begin{array}{l}
\text { there are constants } r>0 \text { and } \rho>0 \text { such that } \Psi(v) \geqslant \rho \\
\text { for every } v \in E \text { with }\|v\|=r,
\end{array}\right. \\
\Psi(0)<\rho \text { and } \Psi\left(v_{0}\right)<\rho \text { for some } v_{0} \in E \text { with }\left\|v_{0}\right\| \geqslant r .
\end{gathered}
$$

Then there is a critical point $v$ of $\Psi$ such that $\Psi(v) \geqslant \rho$.

VERIFICATION OF (PS). We write

$$
K v_{j}+\left|v_{j}\right|^{m^{\prime}-2} v_{j}=w_{j}+\varphi_{j}
$$

with $w_{j} \in L^{m},\left\|w_{j}\right\|_{L^{m}} \rightarrow 0, \varphi_{j} \in N(A) \cap L^{m}$. We have

$$
\begin{gathered}
\frac{1}{2} \int K v_{j} \cdot v_{j}+\frac{1}{2} \int\left|v_{j}\right|^{m^{\prime}}=\frac{1}{2} \int w_{j} v_{j}, \\
\left.\left|\frac{1}{2} \int K v_{j} \cdot v_{j}+\frac{1}{m^{\prime}} \int\right| v_{j}\right|^{m^{\prime}} \mid \leqslant C .
\end{gathered}
$$

Therefore

$$
\left(\frac{1}{m^{\prime}}-\frac{1}{2}\right) \int\left|v_{j}\right|^{m^{\prime}} \leqslant C+\frac{1}{2}\left\|w_{j}\right\|_{L^{m}}\left\|v_{j}\right\|_{L^{m^{\prime}}}
$$

and so $v_{j}$ remains bounded in $L^{m^{\prime}}$. We extract a subsequence-still denoted by $v_{j}$ - which converges weakly to $v$ in $E$. By the convexity of the function $|t|^{m^{\prime}}$ we have

$$
\frac{1}{m^{\prime}}|v|^{m^{\prime}}-\frac{1}{m^{\prime}}\left|v_{j}\right|^{m^{\prime}} \geqslant\left|v_{j}\right|^{m^{\prime}-1} v_{j}\left(v-v_{j}\right)=\left(w_{j}+\varphi_{j}-K v_{j}\right)\left(v-v_{j}\right) .
$$

Thus

$$
\frac{1}{m^{\prime}} \int|v|^{m^{\prime}}-\frac{1}{m^{\prime}} \int\left|v_{j}\right|^{m^{\prime}} \geqslant \int\left(w_{j}-K v_{j}\right)\left(v-v_{j}\right) .
$$

It follows from the compactness of $K$ that the right-hand side of (37) goes to zero and thus $\lim \sup \left\|v_{j}\right\|_{L^{m^{\prime}}} \leqslant\|v\|_{L^{m^{\prime}}}$. Hence $v_{j} \rightarrow v$ strongly in $L^{m^{\prime}}$. 
VERIFICATION OF (35). By (18) we have

$$
\Psi(v) \geqslant-C\|v\|_{L^{1}}^{2}+\frac{1}{m^{\prime}}\|v\|_{L^{m^{\prime}}}^{m^{\prime}} \geqslant-C\|v\|_{L^{m^{\prime}}}^{2}+\frac{1}{m^{\prime}}\|v\|_{L^{m^{\prime}}}^{m^{\prime}}
$$

and the conclusion follows immediately since $m^{\prime}<2$.

VERIFICATION OF (3.6). Choose $v_{0}$ of the form $v_{0}=a v_{1}$ where $v_{1}$ is any element in $E$ such that $\int K v_{1} \cdot v_{1}<0$ and $a$ is large enough.

We now deduce from the Mountain Pass Lemma that there exists $v \in E$, $v \neq 0$, such that $\Psi^{\prime}(v)=0$. Then $u=|v|^{m^{\prime}-2} v$ is a nontrivial solution of the equation $A u+g(u)=0$ with $u \in L^{m}$. Finally we show that $u \in L^{\infty}$ using the same argument as in the proof of Theorem 1 (Step 3).

REMARK 12. Instead of using the Mountain Pass Lemma one can give a very elementary argument. Indeed, minimize $\int K v \cdot v$ on the set $\left\{v \in E ;\|v\|_{L^{m^{\prime}}} \leqslant 1\right\}$. The minimum is achieved at some $v_{0} \in E$ with $\left\|v_{0}\right\|_{L^{m^{\prime}}}=1$ and $\int K v_{0} \cdot v_{0}<0$. Clearly we have $K v_{0}+\lambda\left|v_{0}\right|^{m^{\prime}-2} v_{0}=\varphi$ for some constant $\lambda>0$ and some $\varphi \in N(A) \cap L^{m}$. Then $v=\alpha v_{0}$ is a critical point of $\Psi$ on $E$ provided $\alpha$ is an appropriate constant. Unfortunately this argument relies heavily on the fact that $g(u)$ is homogeneous and does not extend to the general case while the previous argument does.

\section{REFERENCES}

1. H. Amann and G. Mancini, Some applications of monotone operator theory to resonance problems (to appear).

2. H. Amann and E. Zehnder, Nontrivial solutions for a class of nonresonance problems and applications to nonlinear differential equations, Ann. Scuola Norm. Sup. Pisa 8 (1980), 539-603.

3. Multiple periodic solutions for a class of nonlinear autonomous wave equations, Houston $\mathrm{J}$. Math. (to appear).

4. A. Ambrosetti, Recent advances in the study of the existence of periodic orbits of Hamiltonian systems (to appear).

5. A. Ambrosetti and G. Mancini, Solutions of minimal period for a class of convex Hamiltonian systems, Math. Ann. 255 (1981), 405-421.

6. On a theorem of Ekeland and Lasry concerning the number of periodic Hamiltonian trajectories, J. Differential Equations (to appear).

7. A. Ambrosetti and P. Rabinowitz, Dual variational methods in a critical point theory and applications, J. Funct. Anal. 14 (1973), 345-381.

8. A. Bahri, Topological results on a certain class of functionals and applications, J. Funct. Anal. (to appear).

9. A. Bahri and H. Berestytcki, A perturbation method in critical point theory and applications, Trans. Amer. Math. Soc. 267 (1981), 1-32.

10. __ Forced vibrations of superquadratic Hamiltonian systems, Acta Math. (to appear); see also C. R. Acad. Sci. Paris Sér. A 292 (1981), 315-318.

11. Existence of periodic solutions for some second order systems of nonlinear ordinary differential equations, Comm. Pure Appl. Math. (to appear).

12. A. Bahri and H. Brezis, Periodic solutions of a nonlinear wave equation, Proc. Roy. Soc. Edinburgh 85 (1980), 313-320.

13. V. Benci and P. Rabinowitz, Critical point theorems for indefinite functionals, Invent. Math. 52 (1979), 241-273.

14. H. Berestycki and J. M. Lasry, Multiple periodic solutions of Hamilton's equations on a given energy surface (to appear).

15. H. Brezis and J. M. Coron, Periodic solutions of nonlinear wave equations and Hamiltonian systems, Amer. J. Math. 103 (1981), 559-570.

16. H. Brezis, J. M. Coron and L. Nirenberg, Free vibrations for a nonlinear wave equation and a theorem of P. Rabinowitz, Comm. Pure Appl. Math. 33 (1980), 667-689. 
17. H. Brezis and L. Nirenberg, Forced vibrations for a nonlinear wave equation, Comm. Pure Appl. Math. 31 (1978), 1-30.

18. Characterizations of the ranges of some nonlinear operators and applications to boundary value problems, Ann. Scuola Norm. Sup. Pisa 5 (1978), 225-326.

19. F. Browder, Nonlinear elliptic boundary value problems, Bull. Amer. Math. Soc. 69 (1963), $862-874$.

20. D. Campbell, Personal communication and papers to appear.

21. L. Cesari, Existence in the large of periodic solutions of hyperbolic partial differential equations, Arch. Rational Mech. Anal. 20 (1965), 170-190.

22. K. C. Chang, Solutions of asymptotically linear operator equations via Morse theory (to appear).

23. K. C. Chang, S. P. Wu and Shujie Li, Multiple periodic solutions for an asymptotically linear wave equation (to appear).

24. F. Clarke, Periodic solution to Hamiltonian inclusions, J. Differential Equations (to appear).

25. , A classical variational principle for periodic Hamiltonian trajectories, Proc. Amer. Math.

Soc. 76 (1979), 186-188.

26. The dual action, optimal control and generalized gradients (to appear).

27. F. Clarke and I. Ekeland, Hamiltonian trajectories having prescribed minimal period, Comm. Pure Appl. Math. 33 (1980), 103-116.

28. , Nonlinear oscillations and boundary-value problems for Hamiltonian systems, Arch.

Rational Mech. Anal. (to appear).

29. J. M. Coron, Résolution de l'équatin $A u+B u=f$ où $A$ est linéaire et $B$ dérive d'un potentiel convexe, Ann. Fac. Sci. Toulouse 1 (1979), 215-234 and C. R. Acad. Sci. Paris Sér. A 288 (1979), 805-808.

30. Solutions périodiques non triviales d'une équation des ondes, Comm. Partial Differential Equations 6 (1981), 829-848.

31. __ Période minimale pour une corde vibrante de longueur infinie, C. R. Acad. Sci. Paris Sér. A 294 (1982), 127-129.

32. , Periodic solutions of a nonlinear wave equation without assumption of monotonicity (to appear).

33. L. De Simon and G. Torelli, Soluzione periodiche di equazioni a derivate parziali di tipo iperbolico nonlineari, Rend. Sem. Mat. Univ. Padova 40 (1968), 380-401.

34. I. Ekeland, Periodic solutions of Hamiltonian equations and a theorem of P. Rabinowitz, J. Differential Equations 34 (1979), 523-534.

35. __ A perturbation theory near convex Hamiltonian systems (to appear).

36. I. Ekeland and J. M. Lasry, On the number of periodic trajectories for a Hamiltonian flow on a convex energy surface, Ann. of Math. (2) 112 (1980), 283-319.

37. __ Duality in nonconvex variational problem (to appear).

38. J. Hale, Periodic solutions of a class of hyperbolic equations containing a small parameter, Arch. Rational Mech. Anal. 23 (1967), 380-398.

39. $\mathrm{H}$. Hofer, On the range of a wave operator with nonmonotone nonlinearity (to appear).

40. _ A new proof for a result of Ekeland and Lasry concerning the number of periodic Hamiltonian trajectories on a prescribed energy surface (to appear).

41. G. Lamb, Elements of soliton theory, Wiley, New York, 1980.

42. E. Landesman and A. Lazer, Nonlinear perturbations of linear elliptic boundary value problems at resonance, J. Math. Mech. 19 (1970), 609-623.

43. J. Leray and J. L. Lions, Quelques résultat de Visik sur les problèmes élliptiques nonlinéaires par les méthodes de Minty-Browder, Bull. Soc. Math. France 93 (1965), 97-107.

44. H. Lovicarova, Periodic solutions of a weakly nonlinear wave equation in one dimension, Czech.

Math. J. 19 (1969), 324-342.

45. G. Mancini, Periodic solutions of some semilinear autonomous wave equations, Boll. Un. Mat. Ital. 5 (1978), 649-673.

46. J. Mawhin, Solutions périodiques d'équations aux dérivées partielles hyperboliques nonlinéaires, Mélanges Th. Vogel, Presse Univ. Bruxelles, Bruxelles, 1978, pp. 301-319.

47. Compacité, monotonie et convexité dans l'étude de problèmes aux limites semi-linéaires, Lecture Notes Univ. de Sherbrooke, 1981.

48. J. Moser, Periodic orbits near an equilibrium and a theorem by Alan Weinstein, Comm. Pure Appl. Math. 29 (1976), 727-747. 
49. L. Nirenberg, Variational and topological methods in nonlinear problems, Bull. Amer. Math. Soc. (N.S.) 4 (1981), 267-302.

50. H. Poincaré, Les méthodes nouvelles de la mécanique céleste, Gauthier-Villars, Paris, 1982.

51. P. Rabinowitz, Periodic solutions of nonlinear hyperbolic partial differential equations, Comm. Pure Appl. Math. 20 (1967), 145-205.

52. $165-194$

53. ___ Free vibrations for a semilinear wave equation, Comm. Pure Appl. Math. 31 (1978), 31-68.

54. __ Periodic solutions of Hamiltonian systems, Comm. Pure Appl. Math. 31 (1978), 157-184.

55. __ Periodic solutions of a Hamiltonian system on a prescribed energy surface, J. Differential Equations 33 (1979), 336-352.

56. Subharmonic solutions of a forced wave equation, Amer. J. Math. (to appear).

57. _— On nontrivial solutions of a semilinear wave equation, Ann. Scuola Norm. Sup. Pisa (to appear).

58. __ Periodic solutions of Hamiltonian systems: a survey, SIAM J. Math. Anal. (to appear).

59. __ Multiple critical points of perturbed symmetric functionals, Trans. Amer. Math. Soc. 272 (1982), 753-769.

60. M. Struwe, Infinitely many critical points for functionals which are not even and applications to superlinear boundary value problems, Manuscripta Math. 32 (1980), 335-364.

61. J. Toland, A duality principle for nonconvex optimisation and the calculus of variations, Arch. Rational Mech. Anal. 71 (1979), 41-61.

62. __ Duality in nonconvex optimization, J. Math. Anal. Appl. (to appear).

63. O. Vejvoda, Periodic solutions of a linear and weakly nonlinear wave equation in one dimension. I, Czech. Math. J. 14 (1964), 341-382.

64. O. Vejvoda et al., Partial differential equations: time-periodic solutions, Noordhoff, Groningen, 1981.

65. A. Weinstein, Normal modes for a nonlinear Hamiltonian system, Invent. Math. 20 (1973) 47-57.

66. Periodic orbits for convex Hamiltonian systems, Ann. of Math. (2) 108 (1978), 507-518.

67. On the hypotheses of Rabinowitz' periodic orbit theorems, J. Differential Equations 33 (1979), 353-358.

68. M. Willem, Density of the range of potential operators, Proc. Amer. Math. Soc. 83 (1981), $341-344$.

Department of Mathematics, Université de Paris VI, 4Pl. Jussieu, 75230 Paris Cédex 05, France 